\title{
Pattern of Chest Surgery Related Diseases at a Tertiary Care Hospital of South-Punjab, Pakistan
}

\author{
DR. Muhammad Khalid \\ MBBS, NISHTAR HOSPITAL, MULTAN, PAKISTAN. \\ DR. Fatima Bano \\ MBBS, NISHTAR HOSPITAL, MULTAN, PAKISTAN. \\ DR. Anam Paracha \\ MBBS, NISHTAR HOSPITAL, MULTAN, PAKISTAN
}

\begin{abstract}
Objective: To evaluate the pattern of thoracic surgical disease and surgical interventions performed in the Thoracic Surgery Department of a tertiary care hospital of Pakistan.

Methodology: The following is a retrospective descriptive purposive study done in the year 2016 at the Thoracic Surgery Department of the Nishter Medical University Hospital, Multan, Pakistan. All the patients who were admitted through out-patient department or referred from other units, over the year 2016, were analyzed. Variables under consideration included mode of admission, patient's diagnosis, surgical procedure performed, type of anesthesia induced and the mortality. Data was presented using descriptive statistics.

Results: A total of 634 patients (370 females and 264 males) were admitted during the study period, out of which 566 came through the out-patient department while the remaining were referrals from other wards. In total, 697 surgeries were performed with esophagoscopy and dilation being the most frequent (38.7\%), followed by tracheostomy $(16.3 \%)$. Corrosive intake $(49.8 \%)$ accounted as the cause for the highest number of admissions followed by empyema thoracis $(11.4 \%)$.

Conclusion: Local study on the topic of Thoracic Surgery audit, in terms of surgeries performed and variation of cases presented, has become an absolute necessity for better health care planning of Pakistan. The pattern of thoracic surgical diseases varies considerably in different regions and populations. The evaluation of these disease patterns can help to adopt appropriate preventive measures to reduce the incidence of these diseases. Furthermore, it can significantly improve our health system.
\end{abstract}

KEYWORDS: Thoracic surgery, Thoracic surgical diseases, Surgical Audit, Pakistan, Pattern of disease

DOI: $10.7176 / \mathrm{JMPB} / 66-07$

Publication date:June 30th 2020

\section{Introduction}

Surgical audit has been designed for continuous professional development and quality enhancement in the health care system [1]. It involves a critical analysis of the pattern of various surgical diseases, the standard and efficacy of surgical procedures and the associated complications. The auditing process, eventually, results in improved patient's outcome [2] and maintains high-quality health care [3] creating a foundation to modify the various approaches to clinical problems and gives a rationale for local changes in clinical practices. Goveart et al. [4] also highlighted the contribution of auditing in significant reduction of in-hospital cost mainly due to quality improvement. Along with these records, audit also documents clinical details of a patient's mortality [5]. A sound surgical audit exposes the deficiencies in knowledge and skills, hence act as feedback for surgeons to ameliorate their work. A periodic surgical audit also enables to identify pattern and prevalence of different diseases in a specific clinical setting or geographical area. All in all, it presents valuable information about the variations in patient burden, procedures being performed and overall mortality. 
The Thoracic Surgery Department of Nishter Hospital, Multan is an urban tertiary care level hospital which also receives referrals from other departments and various interlinked areas. It is an 18 beds ward along with an operation theatre and an endoscopy suite. The operation theatre runs three times a week and the OPD is conducted once a week. Furthermore, a day is also specified to performing flexible upper gastrointestinal endoscopies.

As Pakistan is a developing country with minimal programs for disease control and prevention, infections like pulmonary tuberculosis are quite common. Smoking plays a major role in adding to the number of chest infections [6]. Moreover, limited resources are a big hindrance to offer quality care to the patients. This has resulted in a massive increase in patient load with thoracic surgical diseases. This situation is bleaker especially in South Punjab and remote areas of Baluchistan [6]. Thus, it has become a need of time to study the prevalence, pattern, and course of different thoracic surgical diseases at the local level, to identify their risk factors and to provide data to policy makers for better planning of future strategies.

\section{Methodology}

This is a retrospective, descriptive cross-sectional study, conducted at Department of Thoracic Surgery, Nishter Hospital Multan, Pakistan for a whole year starting from January 2016 to December 2016 after taking full-fledged consent from the Ethical Board Review at the University. The data for the study were obtained from medical records of the corresponding department and formulated to gather relevant details such as a patient's demographic details, investigations, diagnosis, treatment modalities, and the resultant clinical outcome. Variables such as types of surgical procedures, mode of anesthesia and mortality were also documented. Data were entered into the SPSS (Statistical Package for Social Science) version 20 with results being presented as frequencies and percentages using descriptive statistical tests.

\section{Results}

According to our results, a total of 634 patients were recorded in 2016 in Thoracic Surgical Department among which $89.3 \%(n=566)$ were admitted through OPD while rest of the $10.7 \%(n=68)$ patients were referred from other wards. About $58.3 \%(n=370)$ of the patients were females and $41.6 \%(n=264)$ were males. The mean age of patients was 27 years (range 1 year -85 years). A clinical mortality of 15 patients was recorded too.

About 697 surgical procedure were performed out of which $61.7 \%(n=679)$ were performed under general anesthesia and $38.3 \%(\mathrm{n}=260)$ under local. The most common surgical procedure carried out was esophagoscopy and dilation $(38.7 \%)$ followed by tracheostomy $(16.3 \%)$ and tube thoracostomy $(12.8 \%)$. Further details of performed procedures are presented in Table 1.

Table 1: Details of Surgical Procedures performed

\begin{tabular}{|l|l|l|}
\hline Procedures & Frequency (n) & Percentage (\%) \\
\hline Tracheostomy and & 111 & 16.3 \\
\hline $\begin{array}{l}\text { Esophagoscopy } \\
\text { dilation }\end{array}$ & 263 & 38.7 \\
\hline Colonic interposition & 3 & 0.4 \\
\hline $\begin{array}{l}\text { Bifid stomach tube or iso- } \\
\text { peristaltic stomach tube }\end{array}$ & 13 & 1.9 \\
\hline Lobectomy & 19 & 2.8 \\
\hline Thoracotomy & 19 & 2.8 \\
\hline
\end{tabular}




\begin{tabular}{|l|l|l|}
\hline Esophagectomy & 15 & 2.2 \\
\hline Tube Thoracostomy & 87 & 12.8 \\
\hline Decortication & 16 & 2.4 \\
\hline Rib Resection & 50 & 7.4 \\
\hline Sternotomy & 3 & 0.4 \\
\hline Pleurocutaneous window & 12 & 1.8 \\
\hline Pneumonectomy & 2 & 0.3 \\
\hline Monaldi Procedure & 5 & 0.7 \\
\hline Rigid Bronchoscopy & 10 & 1.5 \\
\hline Rigid Oesophagoscopy & 17 & 2.5 \\
\hline Heller Myotomy & 6 & 0.9 \\
\hline Chamberlain Procedure & 2 & 0.3 \\
\hline $\begin{array}{l}\text { Cervical Lymph node } \\
\text { biopsy }\end{array}$ & 5 & 0.7 \\
\hline $\begin{array}{l}\text { Tracheal Resection and } \\
\text { anastomosis }\end{array}$ & 2 & 0.3 \\
\hline Bullectomy & 2 & 0.3 \\
\hline Open Lung Biopsy & 2 & 0.3 \\
\hline VATS Thymectomy & 3 & 0.6 \\
\hline Chest wall Mass Excision & 4 & $\mathbf{1 0 0}$ \\
\hline Pleural Biopsy & 5 & 0.6 \\
\hline Cardiomyoplasty & 4 & 0.3 \\
\hline VATS Sympathetectomy & 1 & \\
\hline TOTAL & $\mathbf{6 7 9}$ & \\
\hline
\end{tabular}

Over the whole year, a total of 634 cases were registered which required surgical intervention. Among which corrosive intake was most recurring $(49.8 \%)$, followed by empyema thoracis $(11.4 \%)$ and esophageal carcinoma (9.0\%). The least reported cases were of lung carcinoma, mediastinal mass and lung cavitation $(0.3 \%)$. Details of all thoracic surgical cases reported in 2016 are described in Table 2. 
Table 2: Pattern of Thoracic Surgical Cases

Diseases

Corrosive Intake

Post Cricoid Cancer

Esophageal Carcinoma

Lung Cavitation

Pneumothorax

Empyema Thoracis

Mediastinal Mass

Hydropneumothorax

Lung Myectoma

Congenital Lobar Emphysema

Pleural Effusion

Chest wall Mass

Lung Hydatid Cyst

Bronchiectasis

Achalasia

Lung Carcinoma

Hemothorax

Pyopneumothorax

Flail Chest

Laryngeal Carcinoma

Total
Frequency (n)

316

42

57

2

44

72

2

6

3

11

9

11

7

14

13

2

6

6

6

4

634

\section{Percentage (\%)}

49.8

6.6

9.0

0.3

6.9

11.4

0.3

0.9

0.5

1.7

1.4

1.1

2.2

2.1

0.3

0.9

0.9

0.9

0.6

100

\section{Discussion}

Auditing of surgical procedures helps hospitals analyze the outcome and efficiency of a surgical ward. This directly helps to establish a more public accounting for the errors and mishaps that take place during the surgical procedures and eventually confront the morbidity and mortality rates of the patients [7]. In a country like Pakistan lacking resources and funds for managing the outgrowing number of patients presenting to the surgical wards for treatment, auditing can prove to be a blessing.

In our study, tracheostomy was one of the procedures that top the list of the frequently performed procedures. Tracheostomy can be a failure or might result in swallowing and communication disorders after insertion. Emergency dealing physicians in our tertiary care setup still lack the expertise to completely avoid the magnitude of the consequences such a procedure might face and patients habitually return with an aggravated issue. FreemanSanderson et al. [8] highlighted the importance of auditing the procedures of tracheostomy in a tertiary care hospital of Sydney, Australia by collecting a record of 140 patients undergoing the procedure in one year. Regardless of the sizes of the tracheostomy tubes inserted, 109 patients faced speech-language pathologies and were re-admitted, reaffirming the necessity of an audit system. Esophagoscopy and dilation together, were the 
most commonly performed surgical procedures in the thoracic surgery department of our hospital. Around 263 cases reported for the year 2016 which is a humongous number for a single endoscopic procedure in one year. Keren et al [9] worked on a 9-year long retrospective review to audit gastrointestinal endoscopic procedures establishing the fact that endoscopic procedures were a frequent practice in Bnai Zion Medical Center of Haifa, Israel.

Our study also reported corrosives to be a leading cause of emergency procedures similar to a study from Turkey [10] which audited 629 cases in 2 years presenting with a single agent corrosive poisoning. It is worthy of mention that all these upper gastrointestinal endoscopies were performed for the dilation of caustic stricture. In the rural areas of Punjab, prevalence of caustic ingestion was found to be quite high in young females of low socio-economic status [11]. This puts the country under a great financial stress to deal with which compels the government to introduce educational programs and identify the risk factors. Corrosive poisoning in such a big number is in itself alarming for a region and the reason could very well in our country, be the lack of information and education on the harmful effects of poisons and corrosives or even domestic abuse. Dire actions should be taken to find the causes of intentional or forced corrosive ingestion and interventions should be made to prevent fatalities.

Surgeries for esophageal carcinoma were also seen in a considerable number in the Thoracic surgery department. Auditing of this surgery is vital to a hospital's record to analyze whether there has been an increase or decrease in the number of cancer surgeries or their complications over the year that lead to re-admissions. Goan et al. [12] reported cervical anastomotic leakage or pulmonary complications like effusion or empyema to be major reasons for re-admission after esophageal carcinoma surgery. We also present a similar report of approximately 9 cases of pleural effusion and 72 cases of empyema thoracis in our surgical audit study. These can be attributed to a high incidence of infections like tuberculosis in Pakistan where an approximate population of 5.7 million suffer from the aforementioned disease [13]. Only a few patients with lung carcinoma or mediastinal tumors have been recorded in our audit as most of them presented to us at an advanced irresectable stage. This highlights the essence of a routine complete clinical assessment by means of chest X-rays. Periodic screening (annual or semiannual) in the high risk lung carcinoma population with chest X-rays improves the distribution of these patients by disease stage, tumor resectability, and patient survival [13].

There are islands of standardized protocol and work in some of the public health care facilities but the excellence needs to spread to all institutions through active participation and a thorough monitoring process initiated by all professionals. The authors propose a paradigm shift in the pattern of thoracic surgical diseases. Further studies are needed to explore the cases in detail.

\section{Conclusion}

There is an urgent need for country-wide studies on the subject of surgical audit. It should be an obligatory part of every surgeon's training, if not mandatory. The spectrum of diseases needs to be explored and the changes occurring in the pattern of admissions and treatments need to be documented so as to improve health care in Pakistan.

\section{References}

1. Bourke BM, Beiles CB, Thomson IA, Grigg MJ, Fitridge R. Development of the Australasian vascular surgical audit. Journal of vascular surgery. 2012 Jan 1; 55(1):164-9.

2. Beiles CB, Retegan C, Maddern GJ. V ictorian Audit of S urgical M ortality is associated with improved clinical outcomes. ANZ journal of surgery. 2015 Nov; 85(11):803-7.

3. Lui CW, Boyle FM, Wysocki AP, Baker P, D’Souza A, Faint S, Rey-Conde T, North JB. How participation in surgical mortality audit impacts surgical practice. BMC surgery. 2017 Dec;17(1):42.

4. Govaert JA, van Bommel AC, van Dijk WA, van Leersum NJ, Tollenaar RA, Wouters MW. Reducing healthcare costs facilitated by surgical auditing: a systematic review. World journal of surgery. 2015 Jul $1 ; 39(7): 1672-80$.

5. Beiles CB, Retegan C, Maddern GJ. V ictorian A udit of S urgical M ortality is associated with improved clinical outcomes. ANZ journal of surgery. 2015 Nov;85(11):803-7. 
6. Hussain N, Zafar AA. Pattern of Thoracic Surgical Diseases at a Tertiary Care Hospital. Journal of Surgery Pakistan (International). 2015 Jul; 20:3.

7. Dudley H. Necessity for surgical audit. British Medical Journal. 1974 Feb 16;1(5902):275

8. Freeman-Sanderson A, Togher L, Phipps P, Elkins M. A clinical audit of the management of patients with a tracheostomy in an Australian tertiary hospital intensive care unit: Focus on speech-language pathology. International journal of speech-language pathology. 2011 Dec 1;13(6):518-25.

9. Keren D, Rainis T, Stermer E, Lavy A. A nine-year audit of open-access upper gastrointestinal endoscopic procedures: results and experience of a single centre. Canadian Journal of Gastroenterology and Hepatology. 2011;25(2):83-8

10. Avsarogullari L, Senol V, Akdur O, Akin A, Durukan P, Özkan S. Characteristics of acute adult poisonings in a university hospital emergency department in central Turkey: a three-year analysis. JPMA-Journal of the Pakistan Medical Association. 2012 Feb 1; 62(2):129.

11. Hashmi MU, Ali M, Ullah K, Aleem A, Khan IH. Clinico-epidemiological Characteristics of Corrosive Ingestion: A Cross-sectional Study at a Tertiary Care Hospital of Multan, South-Punjab Pakistan

12. Goan YG, Chang HC, Hsu HK, Chou YP. An audit of surgical outcomes of esophageal squamous cell carcinoma. European Journal of Cardio-Thoracic Surgery. 2007 Mar 1; 31(3):536-44.

13. Ali SS, Rabbani F, Siddiqui UN, Zaidi AH, Sophie A, Virani SJ, Younus NA. Tuberculosis: do we know enough? A study of patients and their families in an out-patient hospital setting in Karachi, Pakistan. The International Journal of Tuberculosis and Lung Disease. 2003 Nov 1;7(11):1052-8.

14. Gavelli G, Giampalma E. Sensitivity and specificity of chest X-ray screening for lung cancer. Cancer. 2000 Dec 1; 89(S11):2453-6. 